\title{
Detection of respiratory viruses in cases of porcine respiratory disease in nurseries.
}

\author{
Gerard Martín-Valls ${ }^{1}$, Yanli Li ${ }^{1}$, Ivan Díaz ${ }^{2}$, Esmeralda Cano ${ }^{2}$, Silvana Sosa Portugal ${ }^{1}$, and \\ Enric Mateu ${ }^{1}$ \\ ${ }^{1}$ Universitat Autonoma de Barcelona Facultat de Veterinaria \\ ${ }^{2}$ IRTA-CReSA-UAB edifici CReSA campus UAB 08193 Cerdanyola del Vallès Spain
}

December 30, 2021

\begin{abstract}
Respiratory disease in weaned pigs is a common problem in the field, with a complex aetiology of both viruses and bacteria. In the present study, we investigated the presence of eleven viruses in nasal swabs collected from nurseries (fifty-five clinical outbreaks) under the suspicion of swine influenza A virus (swIAV) by cough and fever. The other ten viruses included influenza $\mathrm{B}$ (IBV) and influenza D viruses (IDV), Porcine reproductive and respiratory syndrome virus (PRRSV), Porcine respiratory coronavirus (PRCV), Porcine cytomegalovirus (PCMV), porcine circoviruses 2 (PCV2), 3 (PCV3) and 4 (PCV), Porcine parainfluenza 1 virus (PPIV1) and Swine orthopneumovirus (SOV). Twenty-nine swIAV-positive cases and twenty-six cases of swIAV-negative respiratory disease were primarily established. IBV, IBD, PCV4 and PPIV1 were not found in any case, while PRCV, SOV, and PCMV were more likely to be found in swIAV-positive nurseries with respiratory disease $(p<0.05)$ although, globally, PCV3, PRRSV, and PCMV were the most frequently detected agents on herd level. At an individual level, the prevalence of different viruses was: swIAV 48.6\%; PRCV 48.0\%; PRRSV 31.6\%; SOV 33.8\%; PCMV 48.3\%, PCV2 36.0\%; and PCV3 33.0\%. Beyond that, it was common to find animals with low Ct values $(<30)$ for all agents except for PCV2 and PCV3. When analysed the association between different pathogens, PRCV was the one with the most associations. It positively interacted $(p<0.05)$ with swIAV and SOV but was negatively associated $(p<0.05)$ with PRRSV and PCVM. Besides these, swIAV and PRRSV were negatively related $(\mathrm{p}<0.05)$. Further analysis of suckling pigs showed that circulation of PRCV, PCMV, SOV, and PCV3 started in the maternities, suggesting a role of the sows in the transmission. Overall, our data may contribute to a better understanding of the complex aetiology and the epidemiology of respiratory disease in weaners. This is the first report of SOV in Spain.
\end{abstract}

Title: Detection of respiratory viruses in cases of porcine respiratory disease in nurseries.

Short title: Respiratory viruses in pig nurseries

Authors: Gerard E. Martín-Valls ${ }^{1^{*}+}$, Yanli Li1 ${ }^{*}$, Ivan Diaz ${ }^{2}$, Esmeralda Cano ${ }^{2}$, Silvana Sosa Portugal ${ }^{1}$, Enric Mateu ${ }^{1,2}$.

1 Dept. de Sanitat i Anatomia Animals, Fac. Veterinaria, Universitat Autonoma de Barcelona, 08193 Cerdanyola del Valles, Spain

2 IRTA-CReSA-UAB, edifici CReSA, campus UAB, 08193 Cerdanyola del Valles, Spain

*Both authors contributed equally

+ Corresponding author

Email: Gerard.martin@uab.cat 
Telephone: +34935812807

Keywords: Respiratory viruses, pig nursery, influenza A, respiratory disease

\section{Summary}

Respiratory disease in weaned pigs is a common problem in the field, with a complex aetiology of both viruses and bacteria. In the present study, we investigated the presence of eleven viruses in nasal swabs collected from nurseries (fifty-five clinical outbreaks) under the suspicion of swine influenza A virus (swIAV) by cough and fever. The other ten viruses included influenza B (IBV) and influenza D viruses (IDV), Porcine reproductive and respiratory syndrome virus(PRRSV), Porcine respiratory coronavirus (PRCV), Porcine cytomegalovirus (PCMV), porcine circoviruses 2 (PCV2), 3 (PCV3) and 4 (PCV), Porcine parainfluenza 1 virus (PPIV1) andSwine orthopneumovirus (SOV). Twenty-nine swIAV-positive cases and twenty-six cases of swIAV-negative respiratory disease were primarily established. IBV, IBD, PCV4 and PPIV1 were not found in any case, while PRCV, SOV, and PCMV were more likely to be found in swIAV-positive nurseries with respiratory disease $(p<0.05)$ although, globally, PCV3, PRRSV, and PCMV were the most frequently detected agents on herd level. At an individual level, the prevalence of different viruses was: swIAV 48.6\%; PRCV 48.0\%; PRRSV 31.6\%; SOV 33.8\%; PCMV 48.3\%, PCV2 36.0\%; and PCV3 33.0\%. Beyond that, it was common to find animals with low Ct values $(<30)$ for all agents except for PCV2 and PCV3. When analysed the association between different pathogens, PRCV was the one with the most associations. It positively interacted $(p<0.05)$ with swIAV and SOV but was negatively associated $(p<0.05)$ with PRRSV and PCVM. Besides these, swIAV and PRRSV were negatively related $(\mathrm{p}<0.05)$. Further analysis of suckling pigs showed that circulation of PRCV, PCMV, SOV, and PCV3 started in the maternities, suggesting a role of the sows in the transmission. Overall, our data may contribute to a better understanding of the complex aetiology and the epidemiology of respiratory disease in weaners. This is the first report of SOV in Spain.

Keywords: porcine respiratory disease complex, swine influenza virus, porcine reproductive and respiratory syndrome virus, swine orthopneumovirus, porcine cytomegalovirus, porcine respiratory coronavirus .

\section{Introduction}

Respiratory disease in nurseries is one of the most common problems of weaned pigs. These processes have complex aetiology involving both viral and bacterial agents. The most common clinical picture of respiratory disease in nurseries is characterized by cough, with or without fever. Nasal discharge, laboured breathing, and increased mortality may be present as well. Since several agents may produce similar lesions, the pathological picture only has an indicative value and do not allow a precise diagnosis. In many nurseries, these respiratory disease outbreaks, are recurrent batch after batch implying an increased use of antimicrobial agents to treat complications .

Viral pathogens are generally considered the primary agents of respiratory disease in nurseries. In the field, several respiratory viruses can circulate simultaneously in nurseries, making it difficult to ascertain the role of each one in the outbreaks and their interactions in a particular case. For some pathogens such as swineInfluenza A virus (swIAV), a primary role in respiratory outbreaks can be assumed despite the variable virulence of different strains. For some other agents, such as the Porcine respiratory coronavirus (PRCV), it is more difficult to establish their role, whether primary or not, in causing respiratory disease (). Additionally, some viral agents, such as Porcine circovirus 2 (PCV2) andPorcine reproductive and respiratory syndrome virus (PRRSV), may have immunosuppressive or immunomodulatory capabilities, impairing or modifying the response to other agents. The immunosuppressive capabilities of Porcine cytomegalovirus (PCMV) have not been fully proven, although PCMV is supposed to regulate the expression of different cytokines (IL-1, IL-2, IL-12, TNF- $\alpha$, and IL-10) .

Bacterial agents can participate in the respiratory disease of weaners as well. Some may be the primary agents, e.g., Mycoplasma hyopneumoniae, while others may act mainly as synergistic agents. The interactions leading to such complications are poorly understood and the evidence available is contradictory 
The scientific consensus indicate that the clinical and pathological picture of the respiratory farm outbreaks will be the result of the interaction of different viruses and bacteria with a host of a given genetic background and immune status under the environmental circumstances of the farm (See for an excellent review).

In the last years, several viruses have been added to the list of potential respiratory pathogens of pigs. Among them, Influenza B (IBV) and D viruses (IDV), swine orthopneumovirus (SOV), Porcine parainfluenza 1 virus (PPIV1) also designated asPorcine respirovirus, and Porcine circovirus 3 (PCV3) are worth mentioning. The knowledge on the epidemiology of these viruses and their participation in respiratory outbreaks is limited with only a few reports available The role of other recently discovered agents such as PCV4 is not known yet .

The present study aimed to explore the participation of several respiratory viruses, including the recently reported SOV, PPIV, PCV3, and PCV4 together with IBV and IDV, in respiratory outbreaks that were initially sent to the laboratory under suspicion of swIAV infection.

\section{Material and methods}

\section{Cases and sample collection}

The study comprised 55 respiratory outbreaks in nurseries (out of the 84 cases received for diagnosis between 2017 and 2019) from which nasal swabs ( $\mathrm{n}=873$, no less than 10 samples per case) were submitted for diagnosis. Outbreaks were defined as situations with noticeable respiratory disease and where predominant signs were cough and fever.

These cases have been previously examined for swIAV during a surveillance of this infection in Spanish farms . The 55 studied outbreaks were comprised of 29 randomly selected outbreaks with swIAV-positive animals and 26 randomly selected swIAV-negative cases. Nasal swab suspensions were pooled ( 3 samples/pool) for the initial analysis of respiratory viruses. For each pathogen found to be present, 10 random positive farms were selected and animas were analysed individually. Each farm was assigned a 1-letter code.

Additionally, since some farms performed routine monitoring of swIAV in farrowing crates and nurseries, we had the opportunity to test the circulation of respiratory viruses at different ages on the same farm in 8 additional cases. This sampling comprised 20 nasal swabs collected in farrowing units and 12 in nurseries. With those numbers, any agent could be detected if present in [?] 15\% or [?]25\% of the animals, farrowing units and nurseries, respectively with $95 \%$ confidence. These samples were examined initially by pooling ( 3 samples/pool).

\section{RNA extraction and RT-PCR}

RNA was extracted using MagMax Core nucleic acid extraction kit (ThermoFisher Scientific) according to the manufacturer's instructions. Presence of the different viruses was examined by real time PCR or real time RT-PCR using the AgPath-ID One-Step RT-PCR Reagents (ThermoFisher Scientific). Detection of swIAV, IBV and IDV, and PCV2, PCV3, and PCV4 was done using the previously described primers. For PPV1, PCMV, PRCV, and SOV, the primers were designed specifically for this study (see table 1). To evaluate the specificity of these primers, positive samples for each one of these viruses were amplified by conventional RT-PCR (see primers in table 1) and sequenced by SANGER sequencing. BLAST was carried out to identify the sequence. The presence of PRRSV was assessed using LSI VetMax PRRS EU/NA v2 reagents. To evaluate farm prevalence with more precision, individual samples from 10 outbreaks positive for each pathogen were also analysed by RT-PCR.

\section{Statistical analysis}

A farm was considered to be infected by a given virus if at least one sample was positive for that virus. The associations between pathogens were tested by means of the $\chi 2$-test considering all other pathogens as potential confounding variables (stratified analysis). In a second step, the probability of two pathogens 
being associated by outbreak was also analysed. Additionally, a k-modes cluster analysis was carried out using Rstudio to evaluate associations of two or more pathogens in respiratory outbreaks.

Individual results from 10 outbreaks were used to determine the prevalence of these viruses in respiratory outbreaks. In this case, average and distribution of Ct-values were also compared by means of Kruskal-Wallis test.

All statistical comparisons using $\chi 2$-test and Kruskal-Wallis were done using the StatsDirect software, and cluster analysis was carried out in $\mathrm{R}$ software, version 4.1.1, using K-modes script.

\section{Results}

Prevalence and association of respiratory viruses PRRSV, PRCV, SoV, PCV2, PCV3, and PCMV in swIAV-positive and -negative nurseries.

The final sampling comprised 55 respiratory farm outbreaks, of which 26 happened in swIAV-positive nurseries. Globally, PCV3 was the most frequently found virus, present in $43 / 55$ outbreaks $\left(78.2 \%\right.$; $\mathrm{CI}_{95 \%}$ : 66.6 87.8\%). PRRSV and PCMV were found in 40/55 outbreaks (72.7\%; $\mathrm{CI}_{95 \%}$ : 58.8-83.5\%); PRCV was found in 29/55 outbreaks $\left(52.7 \%\right.$; $\left.\mathrm{CI}_{95 \%}: 39.3-66.1 \%\right)$; PCV2 was found in $18 / 55$ outbreaks $\left(32.7 \%\right.$; $\mathrm{CI}_{95 \%}: 21.1$ $46.8 \%$ ); and SOV was found in 17/55 outbreaks (30.9\%; $\left.\mathrm{CI}_{95 \%}: 19.5-45.0\right)$. Influenza B, Influenza D, PCV4, and PPIV1 were not detected in any of the outbreaks. To note, this is the first description of SOV occurrence in pig herds in Spain.

Figure 1 shows the proportion of cases in which each pathogen (PRRSV, PRCV, SoV, PCV2, PCV3, PCMV) was found based on the swIAV status of the farm. Interestingly, the frequency of a given pathogen in the swIAV-positive and -negative outbreaks (on a herd level) was different. PRCV, SOV, and PCMV were more likely to be found in swIAV-positive outbreaks $(69.2 \%$ vs. $37.9 \% ; 46.2 \%$ vs. $19.2 \%$ and 88.5 vs. $58.6 \%, p$ $<0.05$, respectively, for the three pathogens considering swIAV-positive versus negative). In the 55 farms, 31 different combinations of the examined viruses were detected (Supplementary table 1 ). In one respiratory outbreak no virus was found.

In the next step, the probability of two pathogens being associated in the outbreaks was analysed. The results (Table 2) suggested that swIAV-positive nurseries were more likely to be infected by PRCV, SOV and PCMV. SOV-infected nurseries were more likely to have PCMV or PRCV-positive animals (Table 2). Moreover, PCMV and PRCV were also statistically associated $(\mathrm{p}<0.05)$. The k-mode cluster analysis indicated that three clusters of outbreaks could be recognized based on the participation of different pathogens: Cluster 1 comprised outbreaks with the presence of swIAV, PRRSV, PRCV and SOV ( $n=7)$, Cluster 2 were cases positive for swIAV, PRRSV, and PRCV, and negative for SOV $(\mathrm{n}=4)$; and Cluster 3 included cases positive for PRRSV and PCMV, and negative for all the other pathogens $(\mathrm{n}=5)$. Globally, the cluster analysis explained just $16 / 55$ cases $(29.1 \%)$.

Prevalence and association of pathogens swIAV, PRRSV, PRCV, SoV, PCV2, PCV3, and PCMV on an individual level

To have further insight on the prevalence of each pathogen, we then performed the analysis at the individual level. Ten batches (one batch of each farm) of infected nurseries for each pathogen were selected; the average $\mathrm{Ct}$ values of each pathogen are shown in Figure 2 and supplementary material 2. As a result, a diversity of Cts (from 15-16 up to 30s) could be found for most of the examined viruses, while it was very uncommon to obtain Ct values of PCV2 and PCV3 below 30. On average, the individual prevalence levels for the different viruses were as follows: swIAV 48.6\%; PRCV 48.0\%; PRRSV 31.6\%; SOV 33.8\%; PCMV 48.3\%, PCV2 $36.0 \%$; PCV3 33.0\%.

The association between different pathogens at the individual level was analysed as well. As shown in Table 3 , PRCV was the pathogen with the most associations. It had positive interactions $(p<0.05)$ with swIAV and SOV but a negative association $(p<0.05)$ with PRRSV and PCVM. Besides these, swIAV and PRRSV were negatively related $(p<0.05)$. 
To examine whether the infection started from the maternal stage, 8 farms where suckling pigs and weaners had been sampled were analysed. In this part only SOV, PCV3, PCMV, and PRCV were included because for swIAV, PRRSV, and PCV2 it is well known that viral circulation may start in maternities. The analysis of the nasal swabs indicated that PCMV spreads mainly in nurseries since the frequency of positive pools in $7 / 8$ farms reached $100 \%$ in that phase (Figure 3). In contrast, for SOV, PCV3, and PRCV the infection was mostly found in suckling pigs, suggesting the role of sows in transmitting the infection.

\section{Discussion}

The polymicrobial nature of the porcine respiratory disease complex is a well-established and widely-accepted concept. Nevertheless, the participation, contribution and interaction between different agents in that complex is not so well understood. One of the main reasons for this, is the difficulty in establishing experimental models that can reflect the complexity of the population in a herd as well as the factors and events that take place in the farm. Moreover, the use of molecular techniques allowed the discovery of new agents. In pigs, the number of viruses known to potentially participate in the respiratory disease has increased with the discovery of IBV, IDV, PPIV1, SOV, PCV3 and PCV4

In the present study, we examined the presence of a panel of 11 viruses in outbreaks of respiratory disease in nurseries. The cases included in this work had been initially submitted for the diagnosis of swIAV because of the clinical signs of the affected animals (cough and fever). Certainly, this could create a selection bias in the sample towards more severe cases of respiratory disease in nurseries. To compensate for the bias of selection based on swIAV suspicion we balanced swIAV-negative and swIAV-positive cases. Moreover, although nasal swabs are optimal samples for those viruses replicating in the nasal epithelium or in the higher airways (such as swIAV or PCMV), its use can underestimate other pathogens, such as PRRSV, in which replication in the nasal mucosa seems to be related to virulence . This bias must be considered as well.

The results of the analysis of pooled nasal swabs indicated that IBV, IDV, PPIV1 and PCV4 were not present in any of the outbreaks. Although from these results it cannot be concluded that these viruses are absent in Spanish pig herds, they suggest that, if present, their frequency is low. This agrees with a previous report .

For the agents present, up to 33 combinations were found. Interestingly, the presence of swIAV in the nurseries was associated with a higher probability that the farm was positive to PRCV, SOV or PCMV. The causes for that association are unknown. It can be speculated that maybe in some farms, these associations reflected a poorer biosecurity or maybe could be the result of a confounding variable. The fact that samples were submitted for diagnosis with limited information makes it impossible to further clarify this point. However, most often the relationship between two different viruses that target the same organs can result in interference. In the present case, when individual results were examined, associations were of different nature.

Animals infected by swIAV were less likely to be infected with PRRSV.In vitro, co-infection of susceptible epithelial CD163 ${ }^{+}$cells with PRRSV and swIAV resulted in interference while, in pigs, some level of interference was observed when PRRSV infection preceded swIAV inoculation . Interestingly, the statistical analysis indicated that swIAV-positive animals were more likely to be infected with PRCV. This result apparently contradicts previous reports indicating that co-infection of swIAV and PRCV resulted in lower viral replication and that prior in vitro infection with swIAV strongly inhibits PRCV replication. In our case, PRCV infection was associated with a lower probability of being infected by PRRSV or PCMV. This observation would be consistent with the known induction of type I interferon by PRCV and the known susceptibility of PRRSV and cytomegaloviruses to interferons. SOV and PRCV seemed to be positively associated. The confirmation of these associations would require either a different epidemiological study or, if possible, an experimental co-infection study.

Another interesting point to discuss is the different distribution of $\mathrm{Ct}$ values between most pathogens and PCV2 and PCV3 (Figure 2). While for the other agents (swIAV, PRRSV, SOV, PCMV), the most common finding in a farm was that nasal swabs could contain either higher or lower viral loads (as deduced from $\mathrm{Ct}$ 
values), most animals yielded Ct values above 30 for PCV2 and PCV3. This fact suggests that shedding of those viruses was relatively low. In the case of PCV2, this is understandable since all animals probably had high levels of maternally derived antibodies and were vaccinated at weaning. For PCV3, the result is more difficult to explain. found a higher prevalence of PCV3 in weaners with severe respiratory disease indicating that Cts about 20-27 were common in diseased animals, while Cts above 30 were found mostly in animals with less severe respiratory disease or asymptomatic. This could be the case in the present study.

Since for some farms we had samples from both suckling piglets and nurseries, we examined the presence of four of the tested viruses (SOV, PCV3, PRCV and PCMV) in those two compartments of the farm. The results clearly indicated that all these agents started to circulate in the farrowing units, suggesting the possibility of transmission from sows in a pattern like in other diseases. For example, in the case of swIAV it is well documented that in endemic farms transmission may start as early as the first week of age .

In summary, the present study shows the complexity of the viruses present in respiratory outbreaks of weaners and suggests the existence of several interactions among them that deserve further study. Moreover, we report here for the first time the presence of SOV in Spain.

\section{Acknowledgements}

This work was partially funded by CEVA Santé Animale (swIAV analysis). Silvana Sosa Portugal was supported by an FIP fellowship of Universitat Autònoma de Barcelona thanks to fundingof CEVA Santé Animale. Our grateful thanks to Gwenaelle Dauphin, Kathrin Lillie-Jaschinski and Stefan Pesch for the critical reading of the manuscript.

\section{Ethics statement}

The authors confirm that the ethical policies of the journal, as noted in the journal's author guidelines page have been adhered to. Samples used in this work were submitted from field veterinarians for diagnostic purposes on the basis of clinical testing. Therefore, according to Directive 63/2010 -Article 1, point 5(b) "non-experimental clinical veterinary practices"- the procedure carried out to obtain data is not within the scope of named directive.

\section{Conflict of interests}

None to declare.

\section{References}

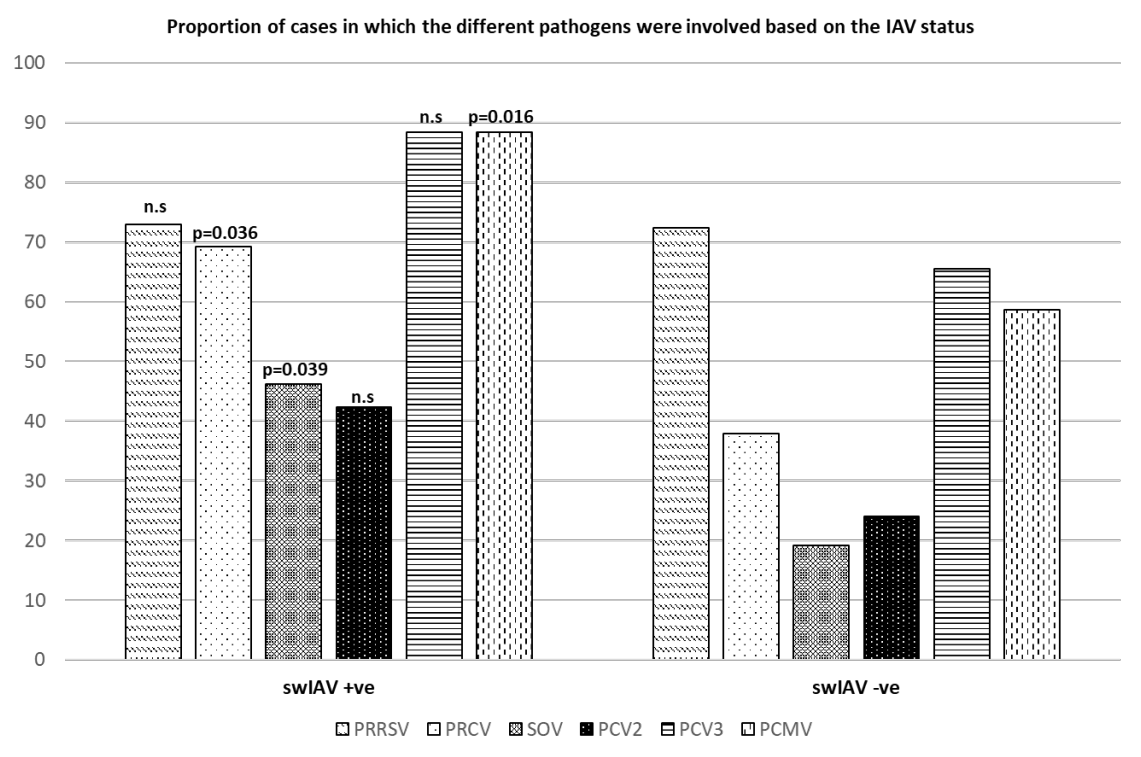


Figure 1. Frequency of detection of the different viruses in the studied outbreaks based on swIAV status of the nursery .

Figure 2. The figure shows the distribution of $\mathrm{Ct}$ values in 10 nurseries infected by each detected virus. To simplify the graphs, values for negative animals were shown as $\mathrm{Ct}=0$. The letter in the $\mathrm{X}$-axis indicates the farm code. Detailed values of prevalence, confidence intervals and average Cts are shown in supplementary material $1 . \mathrm{IAV}=$ influenza $\mathrm{A}$ virus; $\mathrm{PRCV}=$ porcine respiratory coronavirus; $\mathrm{PRRSV}=$ porcine reproductive and respiratory syndrome virus; $\mathrm{SOV}=$ swine orthopneumovirus; $\mathrm{PCMV}=$ porcine cytomegalovirus; PCV2 = porcine circovirus 2 ; PCV3 $=$ porcine circovirus 3 .
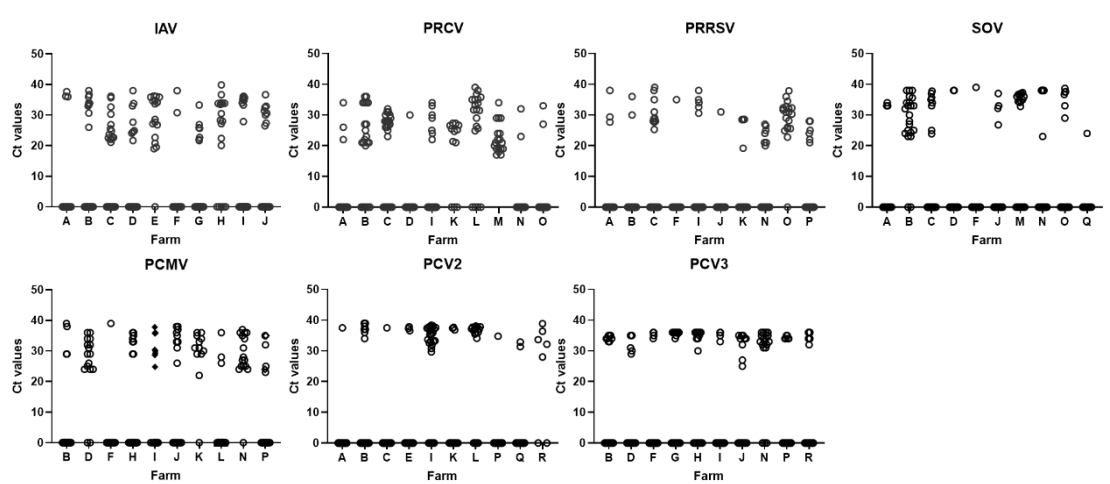

Figure 3. Proportion of positive pools for SOV, PCV3, PCMV and PRCV in suckling pigs and weaners of $\mathbf{8}$ monitored farms. The numbers within the bars indicate the average $\mathrm{Ct}$ value of the positive samples. $\mathrm{PRCV}=$ porcine respiratory coronavirus; $\mathrm{SOV}=$ swine orthopneumovirus; $\mathrm{PCMV}=$ porcine cytomegalovirus; PCV3 = porcine circovirus 3 . 

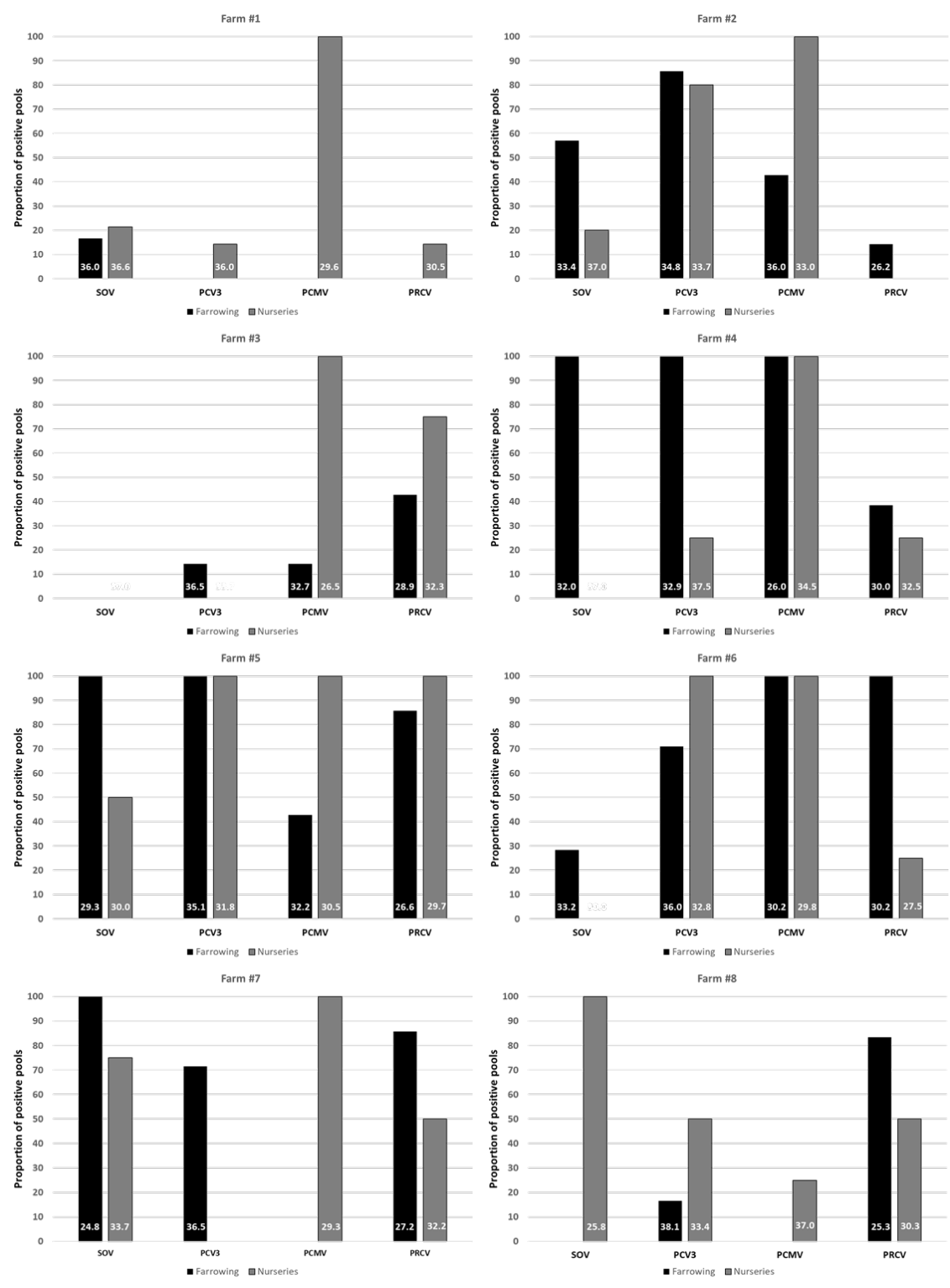

Table 1. List of primers and probes designed for the present study

\begin{tabular}{ll}
\hline Primer & Sequence \\
\hline Real time PCR & \\
$P P V-1 \mathrm{Fw}$ & ATTCGGGCAGAGATGTAACG \\
$P P V-1 \mathrm{Rv}$ & TATCCCACCCCTCGGTCTAT \\
$P P V-1$ Probe & FAM-GCCCGATAAAATCCACAAAGA-BHQ1 \\
$S O V F w$ & GGGGAGGACTTGATGCTGTA \\
$S O V R v$ & AACTTTGCTGCCTCCTTTGA \\
$S O V$ Probe & FAM-CTGAAAGCTGAGAAGGCCAG-BHQ1 \\
$P C M V$ Fw & AATGCGTTTACAACTTCACG \\
$P C M V R v$ & CTGAGCATGTCCCGCCCTAT \\
$P C M V$ Probe & FAM-CTCTAGCGGCGTCCATCACC-BHQ1
\end{tabular}




\begin{tabular}{ll}
\hline Primer & Sequence \\
\hline$P R C V F w$ & TCAGCCAATTTTGGTGACAG \\
$P R C V R v$ & GATCATCCTTTGGCAAGTGG \\
$P R C V$ Probe & FAM-ATGGGAGCAGTGCTAAGCAT-BHQ1 \\
End-point PCR & \\
$P R C V F w$ & AAACACTACTTGTGGTTTTGGTTAT \\
$P R C V R v$ & ACAGTCACACCGAACGGAAT \\
$P C M V F w$ & TGACAGTGAGCAGTCGGAAT \\
$P C M V R v$ & TCAGGCGTGGATATGTAACG \\
$S O V F w$ & CTATCGGAACCGAATGAGAC \\
$S O V R v$ & TGCCAGGAGCCATATTTG \\
\hline
\end{tabular}

Table 2. Statistical associations between pathogens based on the detection in the herd. A nursery was considered to be infected by one of the viruses if at least one animal tested positive for such virus.

\begin{tabular}{lll}
\hline Association & Odds ratio $(\mathbf{9 5 \%} \mathbf{C I})$ & p-value \\
\hline SwIAV-PRCV & $3.68(1.06-13.20)$ & 0.040 \\
SwIAV-SOV & $4.11(1.04-17.75)$ & 0.042 \\
SwIAV-PCMV & $5.41(1.17-33.48)$ & 0.029 \\
SOV-PCMV & N.C.* & 0.002 \\
SOV-PRCV & $18.41(3.23-181.0)$ & $<0.001$ \\
PCMV-PRCV & $13.5(2.39-113.63)$ & 0.001 \\
\hline
\end{tabular}

*All cases where SOV was detected were PCMV-positive.

Table 3. Statistical associations between pathogens based on the detection at individual level. Results were obtained after a stratified analysis of significant one-to-one interactions using the other agents as the stratifying factor. MLE OR = Maximum likelihood Odds Ratio.

\begin{tabular}{llll}
\hline Association & MLE OR & 95\% Confidence Interval & p-value \\
\hline swIAV-PRRSV & 0.209 & $(0.059-0.598)$ & 0.002 \\
SwIAV-PRCV & 2.734 & $(1.164-6.508)$ & 0.011 \\
PRRSV-PRCV & 0.237 & $(0.082-0.604)$ & 0.002 \\
SOV-PRCV & 2.595 & $(1.145-6.059)$ & 0.021 \\
PRCV-PCVM & 0.222 & $(0.085-0.558)$ & 0.002 \\
\hline
\end{tabular}

